\title{
Endoscopic Appearance and Management of Recto-Sigmoid Endometriosis: Case Report
}

\author{
Abubakr Adamª, d, Mechu Narayanan ${ }^{\mathrm{b}}$, Christine Hachem ${ }^{\mathrm{c}}$
}

\begin{abstract}
Endometriosis is the presence of endometrial glands outside the uterine cavity. Endometriosis can involve any part of the bowel but most commonly affects the recto-sigmoid part of the bowel. This case shows the endoscopic views of bowel endometriosis and also elaborates on how to approach and manage similar cases of bowel endometriosis. Patients with bowel endometriosis are better managed and treated with a multidisciplinary team including gastroenterologist, gynecologist and colorectal surgeon.
\end{abstract}

Keywords: Endometriosis; Endoscopy; Histopathology

\section{Introduction}

Endometriosis is the presence of endometrial glands outside the uterine cavity. Endometriosis of the bowel is a form of deep infiltrating endometriosis. The most common location of bowel endometriosis occurs in the recto-sigmoid area. Common presenting symptoms include dysmenorrhea, dyspareunia, cyclic pelvic pain, pain with defecation, constipation, and cyclic rectal bleeding [1].

Diagnosis of intestinal endometriosis can be difficult as it can mimic other diseases. Presentations may be diverse ranging from acute large bowel obstruction to concurrent colorectal cancer [2, 3]. In addition, patients with intestinal endometriosis may present with irritable bowel syndrome (IBS) like symptoms which may be related to the anatomic distribution of the endometriosis as well as an epidemiological association with IBS [4].

The gold standard to diagnose endometriosis is laparoscopy and biopsy to confirm the presence of endometrial

\footnotetext{
Manuscript submitted May 24, 2018, accepted June 20, 2018

aDepartment of Internal Medicine, SSM Health St. Mary's Hospital, St. Louis, MO, USA

${ }^{b}$ Department of Gastroenterology, St. Louis University, St. Louis, MO, USA 'Department of Medicine, Division of Gastroenterology, St. Louis University, St. Louis, MO, USA

${ }^{\mathrm{d} C}$ Corresponding Author: Abubakr Adam, Department of Internal Medicine, SSM Health St. Mary's Hospital, 6420 Clayton Road, St. Louis, MO 63117, USA.Email: dradam999@gmail.com
}

doi: https://doi.org/10.14740/gr1049w glands. As laparoscopy is an invasive procedure, patients with suspected bowel endometriosis should consider non-invasive imaging studies such as transvaginal ultrasound, endoscopic rectal ultrasound or magnetic resonance imaging (MRI) first [5-7]. Colonoscopic examination with biopsy may be helpful in some cases of endometriosis with mucosal and/or submucosal involvement.

\section{Case Report}

We present a 39-year-old woman with past medical history of endometriosis of the umbilicus which was treated with surgery. She presented to the hospital with complaints of deep pelvic pain, dyspareunia, dysmenorrheal pain, cyclical hematochezia and dyschezia for months prior to admission. Patient had suspected pelvic endometriosis and she was started on hormonal therapy and intrauterine contraceptive device without significant improvement.

The patient underwent diagnostic and therapeutic laparoscopy. She was found to have endometriosis like lesions in the cul-de-sac which were removed and sent to histopathology which confirmed presence of endometriosis. The patient also underwent laparoscopic hysterectomy with left-sided oophorectomy and was found to have a mass involving the anterior wall of the recto-sigmoid colon on laparoscopy.

She underwent diagnostic colonoscopy which revealed the presence of an infiltrative partially obstructing large mass in the recto-sigmoid colon (Fig. 1). The mass involved half of the lumen circumference, measured $5 \mathrm{~cm}$ in length and was located $15-20 \mathrm{~cm}$ from the anus. The mass was friable. Biopsies were taken with cold forceps for histology and revealed normal colonic mucosa with features suggestive of a hyperplastic polyp. The remainder of the colon was normal to the terminal ileum.

Patient then underwent trans-rectal endoscopic ultrasound for further characterization of this mass. Endoscopic ultrasound revealed a sub-epithelial lesion extending through deep layers including deep mucosa, muscularis propria, serosa and extending to perirectal fat. Fine needle aspiration (FNA) was performed which showed features suggestive of endometriosis with no features of malignancy. The FNA cytology slide stained positive for CD10 immunostain which stains endometrial cells (Fig. 2).

Patient underwent an elective laparoscopic partial colectomy with re-anastomosis to remove the mass. The resected part of the sigmoid and rectum was sent to histopathology which 


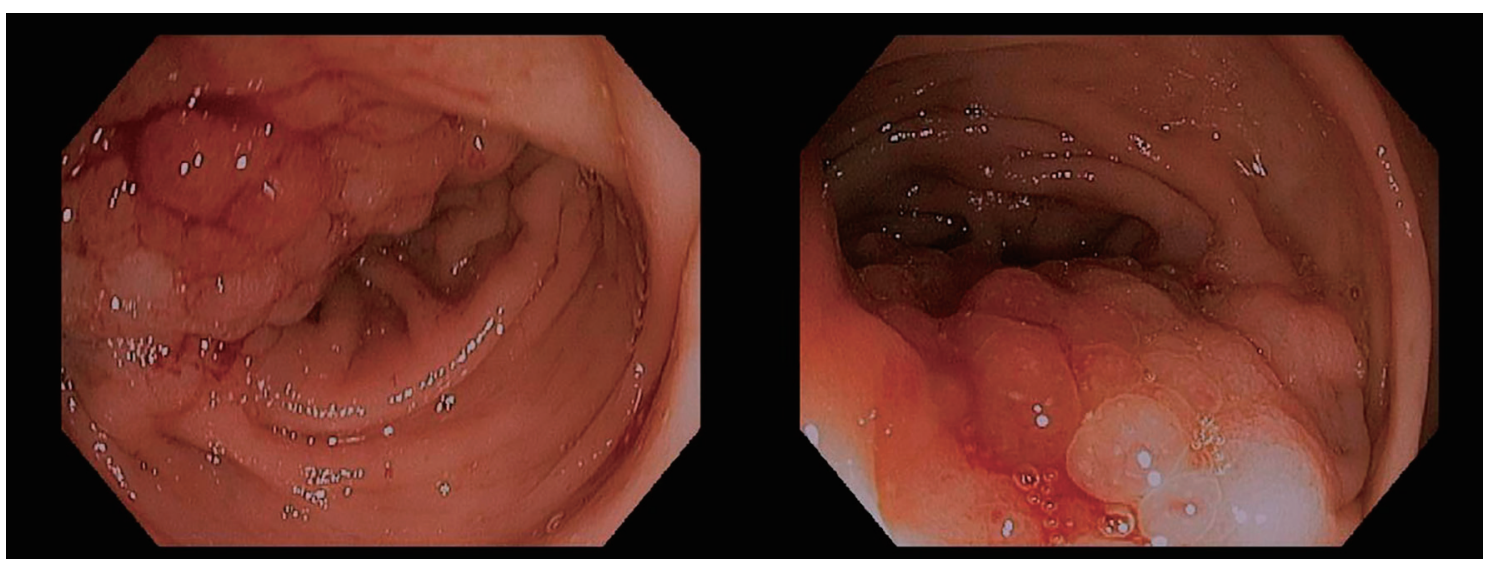

Figure 1. Endoscopic view of recto-sigmoid endometriosis. By courtesy of Dr. Christine Hachem.

revealed the presence of endometrial glands within the bowel wall. Figure 3 reveals the surgical pathology of the resected colon and shows normal looking colonic mucosa to the right of the slide and endometrial glands within the muscularis propria, surrounded with stroma and active bleeding (red blood cells) within the stroma of the glands. Patient was asymptomatic after resection and she was discharged home. She was not discharged on hormonal therapy as she still has an intact right ovary.

\section{Discussion}

Diagnostic evaluation of suspected bowel endometriosis is challenging as it may require an interdisciplinary evaluation including gastroenterologists, gynecologists and colorectal surgeons. MRI in association with CT virtual colonography has been shown to increase the accuracy of the preoperative assessment of colorectal endometriosis [8]. Bowel preparation prior to transvaginal ultrasound has been shown to improve accuracy of diagnosis [5]. Colonoscopy should be an integral part of any evaluation of patients with suspected rectal or co-

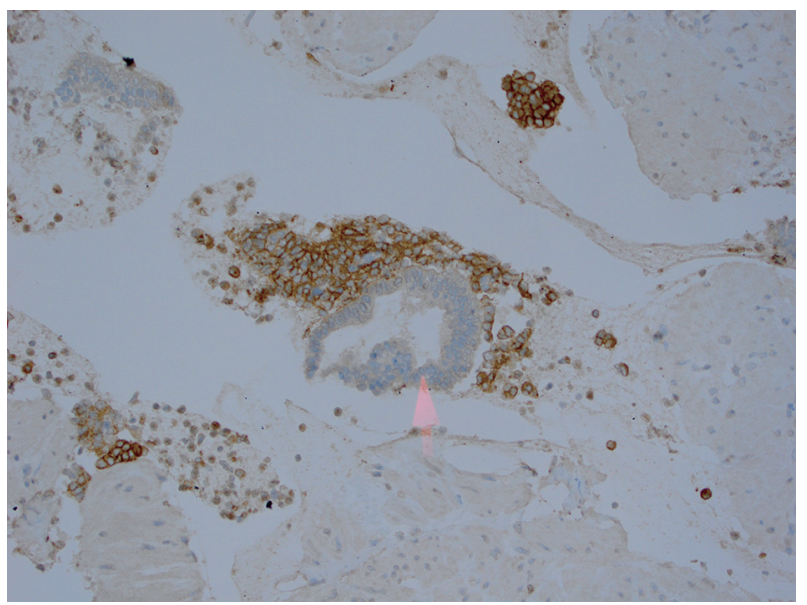

Figure 2. FNA cytology with CD 10 immunostain which stain endometrial cells. By courtesy of Dr. Eric Staros. lonic endometriosis as it may coexist with colorectal carcinoma or may even progress to endometrioid adenocarcinoma $[3,9]$. Negative mucosal biopsies in the setting of high index of suspicion should be further evaluated with rectal ultrasound with FNA given risk of potential false negatives.

Treatment options of bowel endometriosis include both medical and surgical treatments. Most patients with nonobstructive colorectal endometriosis prefer beginning with medical treatment with a low-dose oral contraceptive (OCP) or a progestin [10]. There are three surgical modalities to treat bowel endometriosis and this includes shaving, discoid resection and segmental resection. All three surgical treatment modalities are effective in terms of immediate symptoms relief with comparable complication rates. However, shaving was associated with higher rates of symptom recurrence and reintervention. Data also showed that patients with a nodule $>3$ $\mathrm{cm}$ had a relative risk of 2.5 (95\% CI, 1.66 - 3.99) of requiring bowel resection [11]. Our patient underwent laparoscopic resection as the mass size was $5 \mathrm{~cm}$.

Of the colorectal endometriosis cases, 95\% involve only serosa and muscularis propria; only $6-38 \%$ invade into the mucosa and submucosa, respectively [12]. In the above case, en-

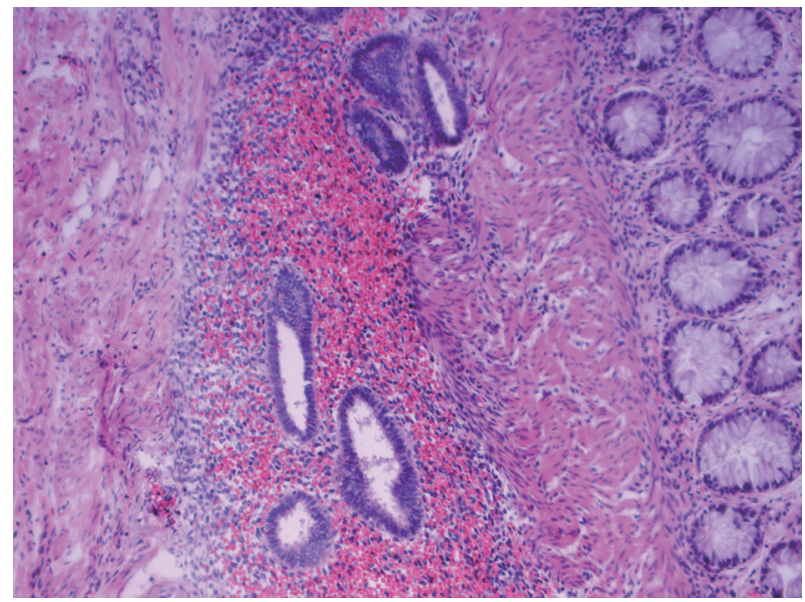

Figure 3. Surgical pathology of the resected colon with endometriosis. By courtesy of Dr. Guihua Cao. 
dometriosis lesions had invaded to the submucosa. Colorectal endometriosis should not be missed as it has a curative treatment either medically or surgically and should be considered in women with rectal bleeding. This case demonstrates the importance of obtaining a gynecological history from women presenting with gastrointestinal tract symptoms, especially regarding association of GI symptoms with menstrual cycles.

\section{Financial Support}

None.

\section{Conflict of Interest}

None.

\section{Consent}

Informed consent was obtained from the patient.

\section{Author Contributions}

All authors participated in preparing and editing the manuscript. The endoscopic images were obtained by the second and third authors. Histopathology image was obtained by the first author.

\section{References}

1. Pereira RM, Zanatta A, Preti CD, de Paula FJ, da Motta EL, Serafini PC. Should the gynecologist perform laparoscopic bowel resection to treat endometriosis? Results over 7 years in 168 patients. J Minim Invasive Gynecol. 2009;16(4):472-479.

2. Allan Z. A case of endometriosis causing acute large bowel obstruction. Int J Surg Case Rep. 2018;42:247249.

3. Ishii M, Yamamoto M, Tanaka K, Asakuma M, Masubuchi S, Hamamoto H, Akutagawa H, et al. Intestinal endometriosis combined with colorectal cancer: a case series.
J Med Case Rep. 2018;12(1):21.

4. Vigano D, Zara F, Usai P. Irritable bowel syndrome and endometriosis: New insights for old diseases. Dig Liver Dis. 2018;50(3):213-219.

5. Ros C, Martinez-Serrano MJ, Rius M, Abrao MS, Munros J, Martinez-Zamora MA, Gracia M, et al. Bowel preparation improves the accuracy of transvaginal ultrasound in the diagnosis of rectosigmoid deep infiltrating endometriosis: a prospective study. J Minim Invasive Gynecol. 2017;24(7):1145-1151.

6. Kim A, Fernandez P, Martin B, Palazzo L, Ribeiro-Parenti L, Walker F, Bucau M, et al. Magnetic resonance imaging compared with rectal endoscopic sonography for the prediction of infiltration depth in colorectal endometriosis. J Minim Invasive Gynecol. 2017;24(7):1218-1226.

7. Scardapane A, Lorusso F, Francavilla M, Bettocchi S, Fascilla FD, Angelelli G, Scioscia M. Magnetic resonance colonography may predict the need for bowel resection in colorectal endometriosis. Biomed Res Int. 2017;2017:5981217.

8. Mehedintu C, Brinduse LA, Bratila E, Monroc M, Lemercier E, Suaud O, Collet-Savoye C, et al. Does computed tomography-based virtual colonoscopy improve the accuracy of preoperative assessment based on magnetic resonance imaging in women managed for colorectal endometriosis? J Minim Invasive Gynecol. 2018.

9. Palla VV, Karaolanis G, Bliona T, Katafigiotis I, Anastasiou I, Hassiakos D. Endometrioid adenocarcinoma arising from colon endometriosis. SAGE Open Med Case Rep. 2017;5:2050313X17745204.

10. Vercellini P, Frattaruolo MP, Rosati R, Dridi D, Roberto A, Mosconi P, De Giorgi O, et al. Medical treatment or surgery for colorectal endometriosis? Results of a shared decision-making approach. Hum Reprod. 2018;33(2):202-211.

11. Afors K, Centini G, Fernandes R, Murtada R, Zupi E, Akladios C, Wattiez A. Segmental and discoid resection are preferential to bowel shaving for medium-term symptomatic relief in patients with bowel endometriosis. J Minim Invasive Gynecol. 2016;23(7):1123-1129.

12. Meuleman C, Tomassetti C, D'Hoore A, Van Cleynenbreugel B, Penninckx F, Vergote I, D'Hooghe T. Surgical treatment of deeply infiltrating endometriosis with colorectal involvement. Hum Reprod Update. 2011;17(3):311326. 